\title{
Use of deep learning in the MRI diagnosis of Chiari malformation type I
}

\author{
Kaishin W. Tanaka ${ }^{1} \cdot$ Carlo Russo $^{1,2} \cdot$ Sidong Liu $^{2,3} \cdot$ Marcus A. Stoodley ${ }^{1} \cdot$ Antonio Di leva $^{1,2}$
}

Received: 26 October 2021 / Accepted: 16 February 2022 / Published online: 24 February 2022

(c) The Author(s) 2022

\begin{abstract}
Purpose To train deep learning convolutional neural network (CNN) models for classification of clinically significant Chiari malformation type I (CM1) on MRI to assist clinicians in diagnosis and decision making.

Methods A retrospective MRI dataset of patients diagnosed with CM1 and healthy individuals with normal brain MRIs from the period January 2010 to May 2020 was used to train ResNet50 and VGG19 CNN models to automatically classify images as CM1 or normal. A total of 101 patients diagnosed with CM1 requiring surgery and 111 patients with normal brain MRIs were included (median age 30 with an interquartile range of 23-43; 81 women with CM1). Isotropic volume transformation, image cropping, skull stripping, and data augmentation were employed to optimize model accuracy. K-fold cross validation was used to calculate sensitivity, specificity, and the area under receiver operating characteristic curve (AUC) for model evaluation.

Results The VGG19 model with data augmentation achieved a sensitivity of $97.1 \%$ and a specificity of $97.4 \%$ with an AUC of 0.99 . The ResNet50 model achieved a sensitivity of $94.0 \%$ and a specificity of $94.4 \%$ with an AUC of 0.98 .

Conclusions VGG19 and ResNet50 CNN models can be trained to automatically detect clinically significant CM1 on MRI with a high sensitivity and specificity. These models have the potential to be developed into clinical support tools in diagnosing CM1.
\end{abstract}

Keywords Artificial intelligence $\cdot$ Chiari I malformation · Convolutional neural network · Deep learning · Magnetic resonance imaging

\section{Abbreviations}

CMI Chiari type I malformation

MRI Magnetic resonance imaging

CNN Convolutional neural network

Kaishin W. Tanaka and Carlo Russo contributed equally to this work.

Antonio Di Ieva

antonio.diieva@mq.edu.au

1 Macquarie Medical School, Macquarie University, NSW 2109 Sydney, Australia

2 Computational NeuroSurgery (CNS) Lab, Macquarie University, Sydney, Australia

3 Centre for Health Informatics, Australian Institute of Health Innovation, Faculty of Medicine, Health and Human Sciences, Macquarie University, Sydney, Australia

\section{Introduction}

Chiari malformations are a group of disorders characterized by anatomical abnormalities of the craniocervical junction (CCJ) with involvement of the cerebellum and brainstem. Chiari malformation type 1 (CM1) is the most common Chiari malformation [1-3]. The precise frequency of CM1 is not known; however, since the advent of MRI, CM1 has been increasingly detected with some studies estimating a prevalence of $1-3.6 \%[4,5]$. The precise natural history of CM1 has not been established and management is generally indicated for patients with severe symptoms, progressive neurological deficits, or those affected by significant syringomyelia with the goal of alleviating symptoms and preventing neurological deterioration [6]. Diagnosis of CM1 rests on identification of displacement of the cerebellar tonsils below the foramen magnum [7]. Currently, it is most widely accepted that a herniation of $5 \mathrm{~mm}$ or more is the minimum criterion for diagnosis of CM1 [8-10]. More recently, there has also 
been recognition of intermediate Chiari subtypes with clinical entities such as the Chiari 0 malformation that is not associated with any cerebellar tonsillar herniation, challenging the widely used $5 \mathrm{~mm}$ cutoff for CM1 [11-15]. There are other radiological findings that are known to be associated with CM1 such as syringomyelia, hydrocephalus, and skeletal anomalies such as platybasia and a hypoplastic posterior cranial fossa[16-18]. As there are no other tissue or blood biomarkers for CM1, diagnosis currently relies on neuroimaging primarily through MRI and identification of the known associated neuroanatomical anomalies.

Artificial Intelligence (AI) techniques have been applied to assist and improve the diagnosis of a number of pathologies across a range of anatomical regions[19] with promising results, especially with regards to pathologies of neurosurgical interest [20]. In particular, the use of machine learning techniques in the application of image-recognition tasks in radiology is a promising emerging technology with the potential to drastically improve the efficiency and ability of radiology in the clinical setting [19]. In recent years, deep learning, an AI technique utilizing convolutional neural networks (CNN), has been shown to yield high sensitivity in automated detection of pathology on medical imaging with a similar (or even higher) performance to medical experts. A supervised learning approach is typically used to train CNNs where end-to-end labelled data is input into the network including cases with a known diagnosis.

Deep learning algorithms and CNNs have been applied in research for radiological diagnosis of a number of pathologies [21-26]; however, current literature on AI applications in CM1 is limited. Urbizu et al. [27, 28] proposed a diagnostic predictive model based on machine learning, identifying and utilizing different anatomical morphometric parameters rather than the standard sole use of the measurement of tonsillar herniation. This is further evidence of the presence of other anatomical factors that could be important in the neuroradiological diagnosis of CM1 which may be too subtle for human detection but could be resolved by means of an AI-based data mining approach.

To our knowledge, there has not been a study applying a deep learning methodology in the automated diagnosis of CM1 in the literature and the present study aims to develop on this with the ultimate goal of expanding the use of AI in CM1 research and clinical applications. A supervised learning approach was utilized with end-to-end labelled MRI images used to train and subsequently test two CNNs. We also tested the effectiveness of different modifications to the $\mathrm{CNN}$ and input data for the optimization of the diagnostic performance. The use of a CNN in the analysis and automated diagnosis of CM1 can open the doors to developing AI-assisted clinical decision tools for controversial areas in the clinical management of CM1 such as management of oligosymptomatic patients, optimization of the surgical technique, and prediction of the clinical outcome and potential for post-operative recurrence.

\section{Methods}

\section{Study participants}

A total of 101 patients who had a diagnosis of CM1 from January 2010 to May 2020 with electronically accessible imaging and who underwent primary surgical intervention at our institution were retrospectively identified; a cohort of 111 patients with normal brain MRIs was also retrieved. Patients had been diagnosed and treated by practicing neurosurgeons and radiologists and as such there were no patients included with possible alternate diagnoses such a spontaneous intracranial hypotension. Normal brain MRIs were defined as individuals with no radiological intracranial pathology as confirmed by certified radiologists (at least 5 years or more experience). To match the CM1 cohort with the normal participants' cohort, the T1 fluid-attenuated inversion recovery (FLAIR) sequence in the sagittal plane was identified as the most consistently obtainable sequence between the two groups. Matching the sequences between the two groups maximized consistency of the resolutions of the images avoiding bias in the training of the $\mathrm{CNN}$ models.

Images were acquired on a $3 \mathrm{~T}$ MR unit (Siemens Magnetom Verio, Erlangen, Germany) with the following parameters: TR/TE/TI 2000/8.4/800 ms; flip angle $150^{\circ}$; field-of-view $240 \times 240 \mathrm{~mm}^{2}$, slice thickness $4.5 \mathrm{~mm}$; matrix $320 \times 320$. All imaging data were saved to secure private servers for the computational analysis. Since 2010, our institute has obtained consent from all patients undergoing treatment for clinical research using their medical data approved by a clinical human research ethics committee. All participants included in this study had signed this consent. This study was approved by the University Human Research Ethics Committee.

\section{MRI data pre-processing}

All MRI volumes were anonymized and transformed to isotropic volumes $\left(1 \mathrm{~mm}^{3}\right.$ voxel size $)$ to homogenize the different spatial resolutions and orientations of the images as the images were acquired from multiple sites. Following the isotropic transformation step, volumes were aligned to a brain atlas using the Insight Toolkit (ITK) library. First, the ITK Multi-resolution Image Registration Method with Multi-resolution Pyramid Image Filter and Versor Rigid 3D Transform Optimizer was used to estimate the rotation, scaling, and offset parameters. Then the ITK Centered Transform was applied using only rotation 
and offset parameters obtained from the Versor Rigid 3D Transform [29]. The co-registration to a brain atlas allowed for automatic selection of a single-central sagittal slice of each initial volume to be used in the CNN models (Fig. 1).

\section{Image datasets}

Four types of image dataset were compared to determine the best image format for the training of the neural networks. The training and testing dataset consisted of full images, cropped images, skull stripped full images, and skull stripped cropped images. The full images were the whole single sagittal images automatically selected after the application of the pre-processing pipeline. The cropped images contained an automatically selected $64 \times 64$ pixel section of the region of interest (coordinates mapped to the reference atlas volume) at the $\mathrm{CCJ}$ where herniation of the cerebellum into the foramen magnum occurs (Fig. 2). The CCJ region was selected automatically as the same image coordinates were utilized for the whole dataset after registration of the MRI volumes to the reference atlas volume. Skull stripped images were derived using the ITK filter "StripTsImageFilter" [30, 31]. All images obtained for all four datasets were normalized to have the same pixel intensity value average as in the ImageNet dataset, thus allowing the use of pre-trained weights in CNN models.

\section{Deep convolutional neural network models}

The models used in this study were ResNet50 and VGG19, the most used CNN models in the biomedical sciences. Both models were initialized with ImageNet pre-trained weights, thus enabling transfer learning, i.e., a model that must be trained on different datasets is "transferred" to a different problem and set of information. This was particularly useful given the small dataset available as transfer learning is proven to speed-up the training steps and improve the accuracy. Since the Chiari image dataset is very different from the ImageNet dataset (that largely consists of images of everyday scenes and not medical imaging), all the layers were trainable. In this way, the initial weights were used to extract the low-level features in the first layers whilst allowing the layers to be updated to better match low-level features of the MRI images, especially in consideration of the fact that no color information is contained in grey scale MRI images.

Each model was trained using the 135 training images and 34 validation images, in one image format selected from the four dataset types that had been derived (i.e., non-skull stripped full image, non-skull stripped cropped image, skull
Fig. 1 Examples of an image cropped to $64 \times 64$ pixels of the craniocervical junction without skull stripping
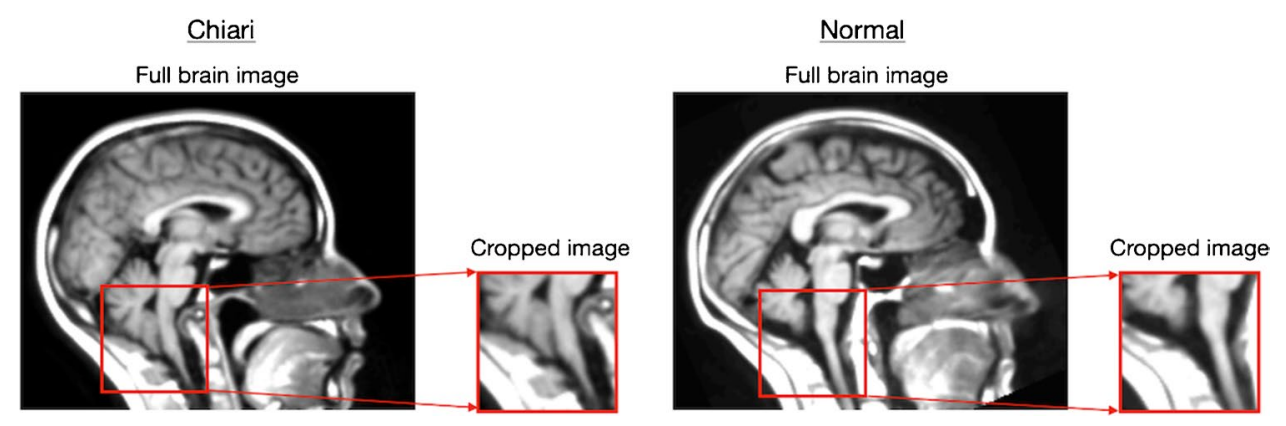

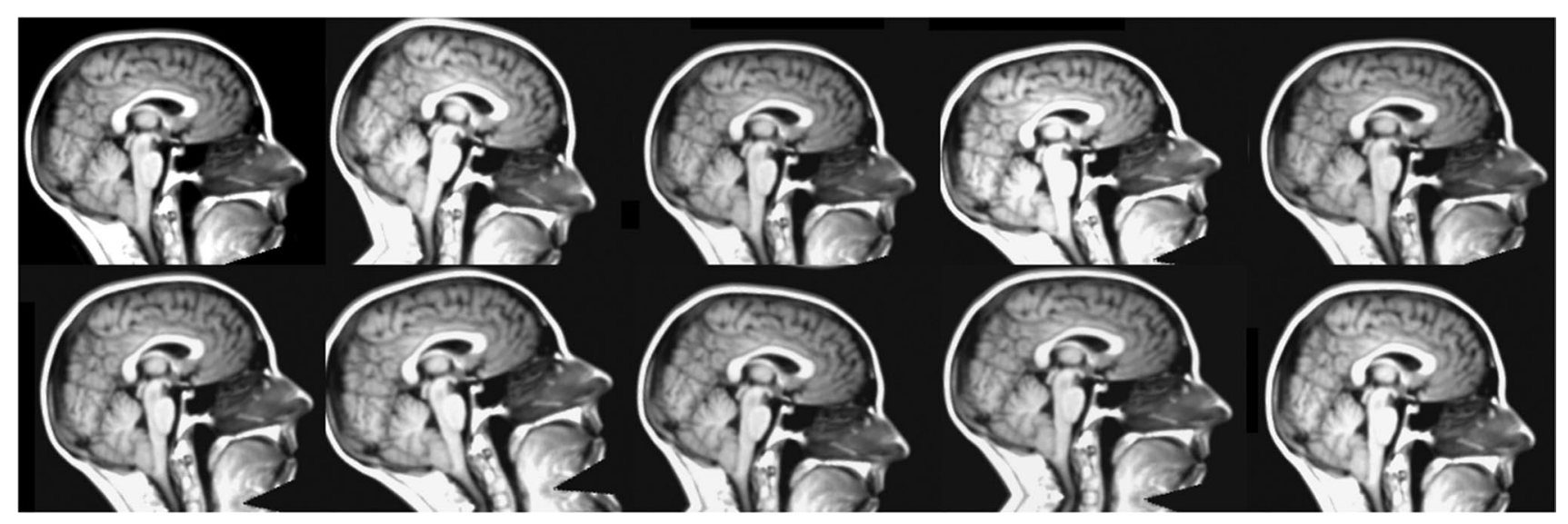

Fig. 2 Examples of images following data augmentation in the starting dataset (original image is in the top left corner) 
stripped full images, and skull stripped cropped images). The number of epochs for each training process was 1000 . Categorical cross entropy was used for the loss function. Only the best model weights were saved after each training epoch based on the value of the validation loss. Batch normalization and a batch size of 16 were used to help reducing over-fitting as well as high dropout rates (0.5 and 0.8$)$ on the last dense layer. The hyperparameters of the training process were fine-tuned through experimentation and it was found that an Adam optimizer with a learning rate of 1e-5 and a weights decay of 1e- 6 gave better results.

\section{Data augmentation}

To improve the training process with a relatively small number of images, the training dataset was augmented by randomly shifting, zooming, rotating, and shearing the initial images. Brightness was also randomly changed on the original training dataset. The parameters used for the data augmentation were selected to emulate more positions and shapes of the cerebellar tonsillar herniation without an excess of deformation. To achieve this, a random rotation range of $15^{\circ}$ clockwise and anticlockwise was performed, 10 pixels for horizontal and vertical random shift, a zoom range of 5\%, shear range of 1.2, and a brightness range from 75 to $130 \%$ of the original pixel intensity value. Figure 2 shows some examples of augmented images starting from one sample.

\section{K-fold cross validation}

To evaluate the performance of the deep learning models, a testing dataset is randomly selected from the whole dataset and held out for evaluation of the performance of the model after it has been trained. Given the small sample size of our dataset, precision of the evaluation of the final accuracy of each model was prone to random bias. Small changes in the measured accuracy were mainly due to the random split of the training and testing dataset. Therefore, the $k$-fold cross validation method was used to estimate the precision of the model's performance where $k$ is an arbitrary number of random partitionings of the initial dataset ( $k=10$ in this study). Within each fold, the dataset was split randomly into the training, validation, and testing subsets, i.e., 43 participants (20\% of the dataset) were held out as a testing dataset; of the remaining 169 participants, 135 (64\% of the dataset) were used for the training of the CNNs and 34 (16\% of the dataset) for validation. A graphical representation of this is seen in Fig. 3. Since the split was random, the ratio between Chiari and Normal participants used for the training was not constant but still balanced for a binary classification task (ranging from a minimum of 56 Chiari cases with 79 normal participants to a maximum of 65 Chiari participants with 70 normal participants).

\section{Results}

Table 1 shows the characteristics of the 101 participants that were included in this study. There was a preponderance of female participants $(80.2 \%)$ which is not unexpected given the well-established increased prevalence of adult CM1 in females[32]. The age of the participants spanned from 5 to 66 years with a median of 30 years (interquartile range from 23-43 years). The age range of the normal participants was from 4 to 93 years (interquartile range from 29-66 years) with 63 females $(56.8 \%)$ and 48 males (43.2\%). The mean cerebellar ectopia was $10.7 \mathrm{~mm}$ with a range extending from 4 to $24 \mathrm{~mm}$. The patient with $4 \mathrm{~mm}$ of ectopia had a band of dura at the CCJ attaching to the cerebellar tonsils with evidence of CSF flow dysfunction at the CCJ on CSF flow studies and characteristic clinical features suggestive of a diagnosis of a CM1 given the underlying pathophysiology. The inclusion of this patient allows for recognition of a spectrum of cerebellar tonsillar herniation in Chiari malformations in the $\mathrm{CNN}$ models.

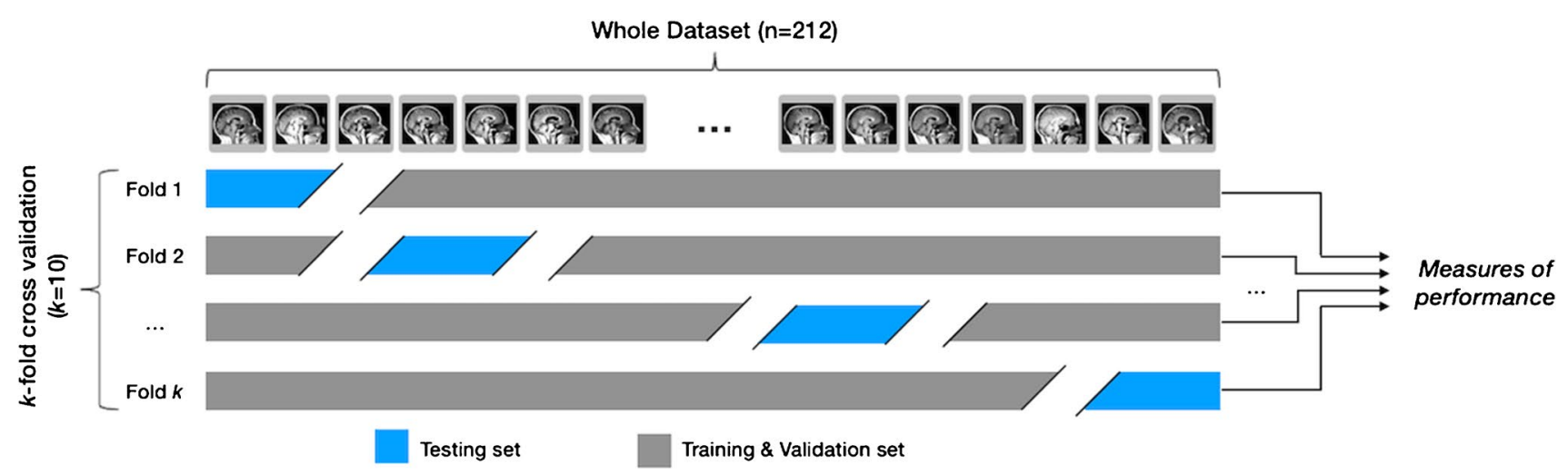

Fig. 3 Graphical representation of the k-fold cross validation technique ( $k=10$ in this study) 
Table 1 Patient characteristics and presenting symptoms

\begin{tabular}{ll}
\hline Characteristic & Value $(N=101)$ \\
\hline Age (years) & \\
Median (interquartile range) & $30(23-43)$ \\
Range & $5-66$ \\
Gender (\%) & \\
Female & $81(80.2)$ \\
Male & $20(19.8)$ \\
Cerebellar ectopia (mm) & \\
Mean (range) & $10.7(4-24)$ \\
Syringomyelia (\%) & \\
Present & $36(35.6)$ \\
Absent & $65(64.4)$ \\
Symptoms (\%) & \\
Headache & $86(85.1)$ \\
Limb pain & $20(19.8)$ \\
Hypoesthesia & $17(16.8)$ \\
Paresthesia & $38(37.6)$ \\
Limb weakness & $10(9.9)$ \\
Vertigo & $20(19.8)$ \\
Nystagmus & $4(4.0)$ \\
\hline
\end{tabular}

Table 2 Measured accuracy of different combinations of convolutional neural networks and dataset settings

\begin{tabular}{|c|c|c|c|}
\hline CNN model & Sensitivity (\%) & Specificity (\%) & AUC \\
\hline \multicolumn{4}{|l|}{ ResNet 50} \\
\hline \multicolumn{4}{|l|}{ Dropout rate 0.5} \\
\hline \multicolumn{4}{|l|}{ Non-cropped images } \\
\hline Non-skull stripped & $86.4 \%$ & $71.4 \%$ & 0.89 \\
\hline Skull stripped & $81.8 \%$ & $90.5 \%$ & 0.93 \\
\hline \multicolumn{4}{|c|}{$64 \times 64$ pixel cropped images } \\
\hline Non-skull stripped & $86.4 \%$ & $95.2 \%$ & 0.98 \\
\hline Skull stripped & $81.8 \%$ & $95.2 \%$ & 0.96 \\
\hline \multicolumn{4}{|l|}{ VGG19 } \\
\hline \multicolumn{4}{|l|}{ Dropout rate 0.5} \\
\hline \multicolumn{4}{|l|}{ Non-cropped images } \\
\hline Non-skull stripped & $86.4 \%$ & $90.5 \%$ & 0.97 \\
\hline Skull stripped & $77.3 \%$ & $100 \%$ & 0.98 \\
\hline \multicolumn{4}{|c|}{$64 \times 64$ pixel cropped images } \\
\hline Non-skull stripped & $86.4 \%$ & $100 \%$ & 1.00 \\
\hline Skull stripped & $77.3 \%$ & $100 \%$ & 0.94 \\
\hline \multicolumn{4}{|l|}{ Dropout rate 0.8} \\
\hline \multicolumn{4}{|l|}{ Non-cropped images } \\
\hline Non-skull stripped & $81.8 \%$ & $100 \%$ & 1.00 \\
\hline Skull stripped & $77.3 \%$ & $100 \%$ & 0.97 \\
\hline \multicolumn{4}{|c|}{$64 \times 64$ pixel cropped images } \\
\hline Non-skull stripped & $95.5 \%$ & $100 \%$ & 1.00 \\
\hline Skull stripped & $72.7 \%$ & $100 \%$ & 0.95 \\
\hline
\end{tabular}

$A U C$ area under receiver operating characteristic curve
Table 2 shows the initial testing of the CNN models that were used in this study with the corresponding dataset types. The ResNet50 model with a dropout rate of 0.5 achieved sensitivities ranging from 81.8 to $86.4 \%$ and specificities ranging from 71.4 to $95.2 \%$ (AUC 0.89-0.97). The VGG19 model with a dropout rate of 0.5 achieved sensitivities ranging from 81.8 to $86.4 \%$ and specificities ranging from 77.3 to $86.4 \%$ (AUC 0.94-0.98). Amongst the two models, the cropped $(64 \times 64$ pixel section of the CCJ $)$ and non-skull stripped datasets were found to have the highest sensitivities and specificities. From this initial testing of different combinations of CNN models and starting image datasets, the VGG19 model with non-skull stripped and cropped dataset images set to a dropout rate of 0.8 was found to achieve the highest sensitivity and specificity of $95.5 \%$ and $100 \%$ respectively (AUC 1.0).

The tenfold cross validation performed on the VGG19 model with the non-skull stripped and cropped dataset images achieved a sensitivity of $98.4 \%$ and a specificity of 94.8\% (AUC 0.99) (Table 3). Similarly, the calculated sensitivity and specificity for the ResNet 50 model (also with a dropout rate of 0.8 ) from tenfold cross validation was $81.2 \%$ and $93.1 \%$ respectively (AUC 0.94 ).

The performance of both models was largely improved by data augmentation. Table 3 shows the tenfold cross validations results on validation and testing dataset for both models with and without data augmentation (using the cropped and non-skull stripped datasets) and Table 4 shows a tenfold cross validation run for VGG19 with optimized pre-processing data settings. Whilst the specificity of the VGG19 model improved with data augmentation from $94.8 \%$ to $97.4 \%$, the sensitivity decreased slightly from 98.4 to $97.1 \%$ (AUC 0.99). Both the sensitivity and specificity of the ResNet 50 model improved to 94.0 and $94.4 \%$ respectively (AUC 0.98).

\section{Discussion}

The application of deep learning CNNs in neuroradiology is an expanding area of research; however its validation in the automated diagnosis of neuroanatomical disorders such as CM1 is limited. This study aimed to apply and validate CNN models in automated diagnosis and identification of clinically significant CM1 to demonstrate the utility of deep learning in developing decision-making support tools for clinicians. We trained VGG19 and ResNet50 CNN models to diagnose CM1 on MRI (utilizing sagittal T1 FLAIR sequences) achieving a high level of sensitivity and specificity as validated with a tenfold cross validation. The CNN models were optimized by utilizing pre-processing image modification techniques including isotropic transformation, region of interest cropping, and data augmentation. The VGG19 model produced the best results with a sensitivity 
Table 3 Comparison between ResNet50 and VGG19 with and without data augmentation (average results using tenfold cross validation)

\begin{tabular}{lllllll}
\hline Model & $\begin{array}{l}\text { Data Aug- } \\
\text { mentation }\end{array}$ & Tenfold validation & Tenfold testing & Sensitivity & Specificity & AUC \\
\hline VGG19 & No & $95.3 \%$ & $96.5 \%$ & $98.4 \%$ & $94.8 \%$ & $99.3 \%$ \\
VGG19 & Yes & $97.4 \%$ & $97.2 \%$ & $97.1 \%$ & $97.4 \%$ & $99.2 \%$ \\
ResNet50 & No & $93.2 \%$ & $86.7 \%$ & $81.2 \%$ & $93.1 \%$ & $94.1 \%$ \\
ResNet50 & Yes & $92.6 \%$ & $94.0 \%$ & $94.0 \%$ & $94.4 \%$ & $98.3 \%$ \\
\hline
\end{tabular}

$A U C$ area under receiver operating characteristic curve
Table 4 Ten-fold cross validation values for CNN model VGG19 with $64 \times 64$ cropped, non-skull stripped datasets with data augmentation achieving the highest accuracy in testing

\begin{tabular}{lcrlll}
\hline Run & Validation & Testing & Sensitivity & Specificity & AUC \\
\hline 1 & $97.1 \%$ & $97.7 \%$ & $100 \%$ & $94.0 \%$ & 1.00 \\
2 & $97.1 \%$ & $100.0 \%$ & $100 \%$ & $100 \%$ & 1.00 \\
3 & $97.1 \%$ & $97.7 \%$ & $100 \%$ & $96.0 \%$ & 0.98 \\
4 & $97.1 \%$ & $95.3 \%$ & $100 \%$ & $94.0 \%$ & 1.00 \\
5 & $94.1 \%$ & $95.3 \%$ & $89.0 \%$ & $100 \%$ & 1.00 \\
6 & $100.0 \%$ & $100.0 \%$ & $100 \%$ & $100 \%$ & 1.00 \\
7 & $100.0 \%$ & $97.7 \%$ & $96.0 \%$ & $100 \%$ & 1.00 \\
8 & $97.1 \%$ & $100.0 \%$ & $100 \%$ & $100 \%$ & 1.00 \\
9 & $94.1 \%$ & $95.3 \%$ & $96.0 \%$ & $95.0 \%$ & 1.00 \\
10 & $100.0 \%$ & $93.0 \%$ & $90.0 \%$ & $96.0 \%$ & 0.94 \\
\hline
\end{tabular}

$A U C$ area under receiver operating characteristic curve

of $97.1 \%$ and specificity of $97.4 \%$ achieved when data augmentation was used (AUC 0.99).

There are limitations in this study that should be considered with the interpretation of the results. The dataset of 212 participants was small and limited to a single center, which limited the case numbers that were usable in the training, testing, and validation subsets and thus performing unsupervised learning to look for clustering of neuroanatomical features was unable to be performed. The small dataset size also prevented an analysis of unilateral or asymmetrical CM1 as the inclusion of stacks of images lateral to the midline sagittal image in the pre-processing pipeline would introduce a degree of variability necessitating a larger number of participants. The random splitting of the data for the cross validation also represented a potential source of random error; however, this was circumvented by use of the tenfold cross validation method with data augmentation. Thus, the final sensitivity and specificity rates achieved by the CNN models in this study were still high (97.2\% for VGG19).

This study was also limited by the MRI sequence availability as we were restricted to using sagittal T1 MRI sequences for consistency between both the CM1 patient dataset and the normal brain dataset. This is partly due to the small sample size necessitating a more uniformly consistent dataset to minimize any bias in the training of the $\mathrm{CNN}$

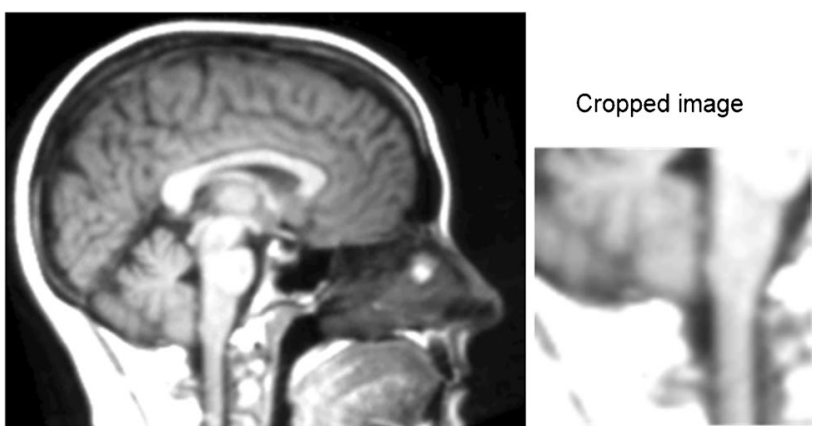

Fig. 4 Example of a normal MRI misclassified as Chiari

models. Amongst the datasets collected for this study, the T1 sagittal FLAIR sequence was most consistently available between CM1 and normal participants. Although an isotropic transformation was applied to the image datasets, the use of a single type of MRI sequence and anatomical plane represents a further restriction to the generalizability of this study.

The highest sensitivity and specificity were achieved in the VGG 19 model with the $64 \times 64$ cropped and non-skull stripped data augmented images (Table 2). Skull stripping with the ITK filter was found to crop out pertinent features at the CCJ (such as the tonsillar herniation) and hence demonstrated inferior performance. The original rationale for testing skull-stripped images was to further enhance the accuracy of the models by eliminating regions of the head that are far removed from the region of interest. Skull stripping with the ITK filter was found to also crop out pertinent features at the CCJ (such as the tonsillar herniation) and hence demonstrated inferior performance. This is not surprising as it known that anatomical differences in the skull base or cranial vault are known to be important factors in the pathogenesis of CM1 [33, 34]. This highlights a need for new techniques that can incorporate the spinal content with the exclusion of the surrounding bone to improve the currently available skull stripping methodologies.

A review of the misclassified cases revealed that they were borderline cases of CM1 with evidence of crowding at the CCJ without overt tonsillar herniation on MRI. Figure 4 shows an example of a normal patient MRI misclassified as 
CM1 by the models. Although there is no significant tonsillar herniation seen, there is suggestion of cerebellar crowding at the CCJ which we suspect is the reason for the misclassification. Similarly, in Fig. 5, a CM1 patient was misclassified as normal with a similar appearance to the posterior fossa with no clear tonsillar herniation. In this case, a combination of the clinical history and review of the $\mathrm{T} 2$ sequence is what lead to the diagnosis of CM. T2 sequences allow for better delineation of cerebellar tonsillar herniation and posterior fossa crowding and would have been the preferred MRI sequence in this study although this sequence was not readily available between the CM1 and control groups. It is not surprising that the CNN model misclassified this case in the absence of clinical data given the equivocal appearance of the posterior fossa on the $\mathrm{T} 1$ sequence. The fact that the misclassified cases were borderline lends weight to the validity of the trained CNN model as the misclassifications in the models only arose in truly equivocal cases.

The results of this study represent the first step towards development of an AI model that can assist clinicians in recognizing clinically significant CM1 and has potential to be developed into a tool for diagnosis, surgical outcome prognostication, developing optimal surgical techniques, and guiding management of recurrence. Clinical studies on these problems are difficult to execute and there are no highly powered studies to guide clinicians. The current VGG19 model could be augmented by incorporating clinical outcome data, post-operative imaging, and post-operative outcomes that are matched to the input images to create a multifaceted clinical support tool. This data can be collated and unsupervised learning analyses using the models can be used to identify clustering of pertinent radiological and clinical features to further elucidate the underlying pathophysiology of disrupted cerebrospinal fluid circulation in CM1. The diagnostic scope of this model could also be further enhanced by including datasets of other structural pathological entities to train the $\mathrm{CNN}$ to allow automated diagnosis of a range of other neuroanatomical anomalies.

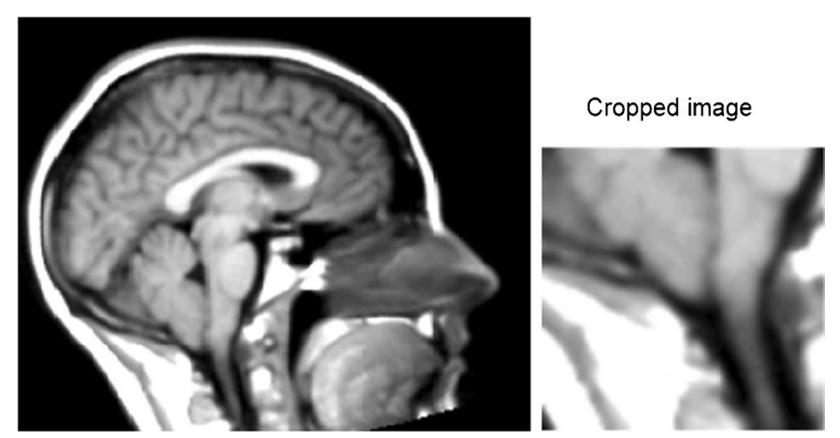

Fig. 5 Example of a Chiari MRI misclassified as normal

\section{Conclusion}

In conclusion, the high sensitivity and specificity rates achieved in these CNN models represents a successful approach to automated recognition of clinically significant CM1 in MRI images. Whilst there are some limitations to the generalizability of the study, these results represent a starting point for further AI assisted research of CM1. Further studies including larger sample sizes, post-operative imaging, and clinical data are needed to improve the accuracy and generalizability of the models and to work towards developing a clinical support tool for managing CM1.

\section{Funding infromation}

The authors received no financial support for the research and authorship of this article.

Supplementary Information The online version contains supplementary material available at https://doi.org/10.1007/s00234-022-02921-0.

Funding Open Access funding enabled and organized by CAUL and its Member Institutions.

\section{Declarations}

Conflict of interest We declare that we have no conflict of interest.

Ethical approval This study was approved by the Macquarie University Human Research Ethics Committee.

Informed consent All participants included in this study had provided signed consent for participation.

Open Access This article is licensed under a Creative Commons Attribution 4.0 International License, which permits use, sharing, adaptation, distribution and reproduction in any medium or format, as long as you give appropriate credit to the original author(s) and the source, provide a link to the Creative Commons licence, and indicate if changes were made. The images or other third party material in this article are included in the article's Creative Commons licence, unless indicated otherwise in a credit line to the material. If material is not included in the article's Creative Commons licence and your intended use is not permitted by statutory regulation or exceeds the permitted use, you will need to obtain permission directly from the copyright holder. To view a copy of this licence, visit http://creativecommons.org/licenses/by/4.0/.

\section{References}

1. Kahn EN, Muraszko KM, Maher CO (2015) Prevalence of Chiari I malformation and syringomyelia. Neurosurg Clin N Am 26:501-507 
2. Heiss JD, Argersinger DP (2020) Epidemiology of Chiari I malformation. In: Tubbs RS, Turgut M, Oakes WJ (eds) The Chiari Malformations. Springer International Publishing, Cham, pp 263-274

3. Sadler B, Kuensting T, Strahle J et al (2020) Prevalence and impact of underlying diagnosis and comorbidities on Chiari 1 malformation. Pediatr Neurol 106:32-37

4. Speer MC, Enterline DS, Mehltretter L et al (2003) Review article: Chiari type I malformation with or without syringomyelia: prevalence and genetics. J Genet Couns 12:297-311

5. Strahle J, Muraszko KM, Kapurch J et al (2011) Chiari malformation Type I and syrinx in children undergoing magnetic resonance imaging. J Neurosurg Pediatr 8:205-213

6. Novegno F, Caldarelli M, Massa A et al (2008) The natural history of the Chiari Type I anomaly. J Neurosurg Pediatr 2:179-187

7. Piper RJ, Pike M, Harrington R et al (2019) Chiari malformations: principles of diagnosis and management. BMJ 365:11159

8. Caldarelli M, Di Rocco C (2004) Diagnosis of Chiari I malformation and related syringomyelia: radiological and neurophysiological studies. Child's Nervous System 20:332-335

9. Aboulezz AO, Sartor K, Geyer CA et al (1985) Position of cerebellar tonsils in the normal population and in patients with Chiari malformation: a quantitative approach with MR imaging. J Comput Assist Tomogr 9:1033-1036

10. Pillay PK, Awad IA, Little JR et al (1991) Symptomatic Chiari malformation in adults: a new classification based on magnetic resonance imaging with clinical and prognostic significance. Neurosurgery 28:639-645

11. Tubbs RS, Elton S, Grabb P et al (2001) Analysis of the posterior fossa in children with the Chiari 0 malformation. Neurosurgery 48:1050-1054 (discussion 1054-1055)

12. Zhou Y, Wang H, Li N et al (2016) Chiari 0 malformation with syringomyelia syringobulbia and syrinx cavity in pons. Interdisciplinary Neurosurgery 6:35-37

13. Schijman E (2004) History, anatomic forms, and pathogenesis of Chiari I malformations. Child's nervous system : ChNS : official journal of the International Society for Pediatric Neurosurgery 20:323-328

14. Lawrence BJ, Urbizu A, Allen PA et al (2018) Cerebellar tonsil ectopia measurement in type I Chiari malformation patients show poor inter-operator reliability. Fluids and Barriers of the CNS $15: 33$

15. Isik N, Elmaci I, Kaksi M et al (2011) A new entity: Chiari Zero malformation and its surgical method. Turk Neurosurg 21:264-268

16. Elster AD, Chen MY (1992) Chiari I malformations: clinical and radiologic reappraisal. Radiology 183:347-353

17. Brito J, BaD Santos, Nascimento IF et al (2019) Basilar invagination associated with chiari malformation type I: A literature review. Clinics (San Paulo, Brazil) 74:e653

18. Milhorat TH, Nishikawa M, Kula RW et al (2010) Mechanisms of cerebellar tonsil herniation in patients with Chiari malformations as guide to clinical management. Acta Neurochir (Wien) 152:1117-1127

19. Hosny A, Parmar C, Quackenbush J et al (2018) Artificial intelligence in radiology. Nat Rev Cancer 18:500-510
20. Senders JT, Arnaout O, Karhade AV et al (2018) Natural and artificial intelligence in neurosurgery: a systematic review. Neurosurgery 83:181-192

21. Kaka H, Zhang E, Khan N (2021) Artificial intelligence and deep learning in neuroradiology: exploring the new frontier. Can Assoc Radiol J 72:35-44

22. Jian A, Jang K, Manuguerra $M$ et al (2021) Machine learning for the prediction of molecular markers in glioma on magnetic resonance imaging: a systematic review and meta-analysis. Neurosurgery 89:31-44

23. Di Ieva A, Russo C, Liu S et al (2021) Application of deep learning for automatic segmentation of brain tumors on magnetic resonance imaging: a heuristic approach in the clinical scenario. Neuroradiology 63:1253-1262

24. Gao Y, Xiao X, Han B et al (2020) Deep Learning methodology for differentiating glioma recurrence from radiation necrosis using multimodal magnetic resonance imaging: algorithm development and validation. JMIR Medical Informatics 8:e19805

25. Liu S, Shah Z, Sav A et al (2020) Isocitrate dehydrogenase (IDH) status prediction in histopathology images of gliomas using deep learning. Sci Rep 10:7733

26. Dai X, Huang L, Qian Y et al (2020) Deep learning for automated cerebral aneurysm detection on computed tomography images. Int J Comput Assist Radiol Surg 15:715-723

27. Urbizu A, Martin BA, Moncho D et al (2018) Machine learning applied to neuroimaging for diagnosis of adult classic Chiari malformation: role of the basion as a key morphometric indicator. $\mathrm{J}$ Neurosurg 129:779-791

28. Urbizu A, Martin BA, Moncho D et al (2017) Machine learning applied to neuroimaging for diagnosis of adult classic Chiari malformation: role of the basion as a key morphometric indicator. $\mathrm{J}$ Neurosurg 129:779

29. Yoo TS, Ackerman MJ, Lorensen WE et al (2002) Engineering and algorithm design for an image processing Api: a technical report on ITK-the Insight Toolkit. Studies in health technology and informatics 85:586-592

30. Bauer SFT, Reyes M (2013) A Skull-Stripping Filter for ITK. Insight Journal. https://doi.org/10.5281/zenodo.811812

31. Mccormick M, Liu X, Jomier J et al (2014) ITK: enabling reproducible research and open science. Front Neuroinform 8:13

32. Marty P, Urbizu A, Macaya A et al (2015) Gender-specific differences in adult type I Chiari malformation morphometrics (P4174). Neurology 84:P4.174

33. Diniz JM, Botelho RV (2020) The role of clivus length and cranial base flexion angle in basilar invagination and Chiari malformation pathophysiology. Neurol Sci 41:1751-1757

34. Heiss JD, Patronas N, Devroom HL et al (1999) Elucidating the pathophysiology of syringomyelia. J Neurosurg 91:553-562

Publisher's Note Springer Nature remains neutral with regard to jurisdictional claims in published maps and institutional affiliations. 